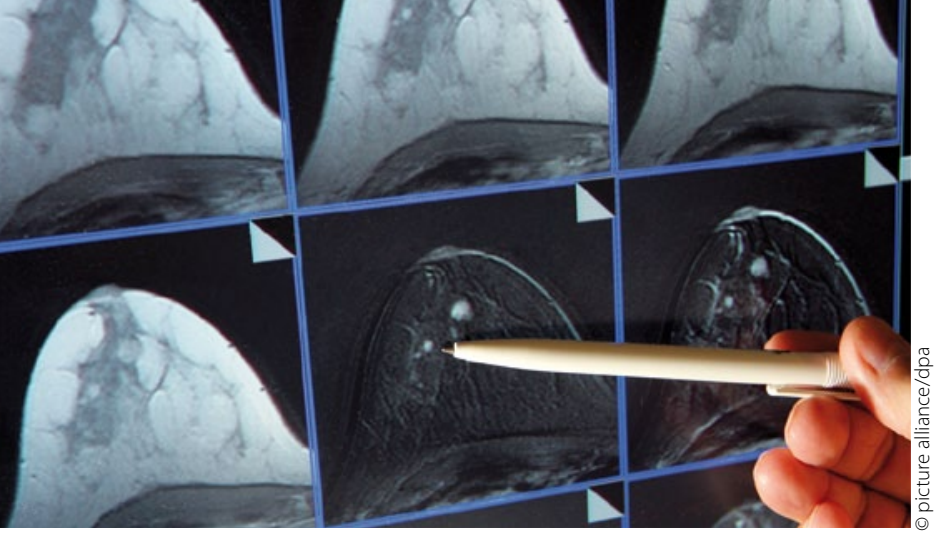

Lebensrettende Früherkennung vs. Überdiagnostik.

EUROSCREEN-Studie

\title{
Bilanz zum Brustkrebs-Screening: Der Nutzen überwiegt!
}

Der Nutzen eines Brustkrebs-Screenings überwiegt die Nachteile, die den Frauen durch Überdiagnostik und falsch-positive Resultate entstehen. Das ist die Bilanz der EUROSCREENArbeitsgruppe nach Analyse zahlreicher europäischer Screening-Programme.

- Auf 1000 Frauen, die vom 50. Lebensjahr an zweimal jährlich über etwa 20 Jahre gescreent werden, kommen sieben bis neun Fälle, in denen der Tod durch Brustkrebs verhindert wird. Demgegenüber stehen vier Fälle von Überdiagnostik, also Frauen, bei denen der Brustkrebs ohne Screening zu Lebzeiten nie diagnostiziert worden wäre.

Das Brustkrebsrisiko ohne bevölkerungsbasiertes Screening liegt für Frauen zwischen 50 und 79 Jahren im Schnitt bei 6,7\%. Die Mortalität beträgt unter diesen Voraussetzungen $3 \%$. Bei 67 von 1000 Frauen wird also Brustkrebs diagnostiziert, 30 von ihnen sterben daran. Wo ein Screening eingeführt wurde, ging die Brustkrebsmortalität deutlich zurück; so starben von 1000 gescreenten Frauen innerhalb von 30 Jahren nur noch maximal 23. In Deutschland werden Frauen zwischen 50 und 69 alle zwei Jahre schriftlich zur Teilnahme am Mammografie-Screening gebeten. Dabei ist die Möglichkeit, unnötig mit einem Krebsverdacht konfrontiert zu werden, für viele Frauen ein Argument gegen die Teilnahme. Wie die EUROSCREEN-Experten errechneten, müsste man bei nicht invasiver Diagnostik in $17 \%$ mit falsch positiven Resultaten rechnen. Bei Einsatz invasiver Verfahren wie der Biopsie beträgt diese Risiko jedoch nur noch $3 \%$. Von 1000 gescreenten Frauen würden also im einen Fall 170, im anderen 30 mindestens einmal wegen eines verdächtigen Befundes wiedereinbestellt, um dann zu erfahren, dass das Ergebnis letztlich negativ ist. Dieser psychischen Belastung wären die Frauen aber jeweils nur kurzzeitig ausgesetzt, betonen die Autoren um Prof. Eugenio Paci, Florenz.

Letztlich sei die Chance, durch ein bevölkerungsbasiertes Brustkrebsscreening Leben zu retten, größer als das Risiko einer Überdiagnostik. Der Benefit im Hinblick auf die Mortalität sei wahrscheinlich sogar größer, als man bisher angenommen hat. Dies müsse man den durch die vielen Diskussionen verunsicherten Frauen hinreichend kommunizieren.

EO - 